\title{
Examining XMM Observations Made in the Galactic Bulge Survey Region
}

\author{
Vicente Estrada-Carpenter ${ }^{1}$ \\ Southwestern University \\ 1001 E. University Ave, Georgetown TX 78626, USA \\ E-mail:vestrada78840@gmail.com

\section{Dr. Robert Hynes} \\ Louisiana State University \\ 112 Thomas Boyd Hall, Baton Rouge, LA 70803, USA \\ E-mail:rih@phys.lsu.edu
}

\begin{abstract}
An X-ray catalog (VXMM) was created to find low mass X-ray binaries (LMXBs) in the Galactic Bulge Survey (GBS). VXMM consists of XMM-Newton detections made in the GBS region, two 6x1 degree regions located 1 degree above and below the Galactic plane respectively. XMM data were downloaded from NASA's database. Source detection was conducted on filtered data sets, using the 2XMM Serendipitous Survey as a guideline for the procedure but incorporating data more recent than 2XMM. Detected sources make up the VXMM catalog, which was used to cross reference with the GBS catalog to find GBS sources in the XMM data. The VXMM catalog contains 107 GBS sources also detected by XMM. The spectra of several of these sources were examined to see which could be classified based on the XMM data. Here we focus on CX13, the brightest unclassified GBS source detected. CX13 was determined not to be an active star as its temperature would be too high. Using a power-law model fit an LMXB was ruled out, as was a background AGN after the variability power spectrum was analyzed. The most likely remaining interpretation of its X-ray spectrum and variability is that it is an absorbed magnetic cataclysmic variable. For comparison, two other GBS sources (CX5 and CX2) are presented.
\end{abstract}

Frank N. Bash Symposium 2013: New Horizons in Astronomy (BASH 2013)

October 6-8, 2013

Austin, Texas

Speaker 


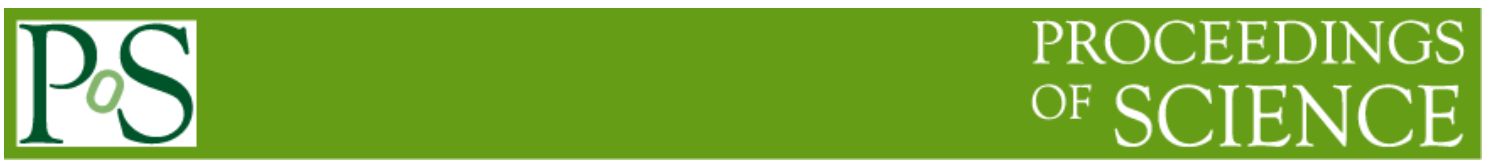

\section{Cons tructing the VXMM Catalog}

The VXMM catalog consists of observations that contributed to the 2XMM catalog and ones obtained afterwards. To build the catalog, the guidelines used by 2XMM were followed. Source detection was run on all available data sets EMOS1, EMOS2, and EPN simultaneously. A total of 30 observations were examined and 1213 sources detected. Once the VXMM and GBS were cross referenced, 107 matches were found. Source locations were found with a $95 \%$ confidence.

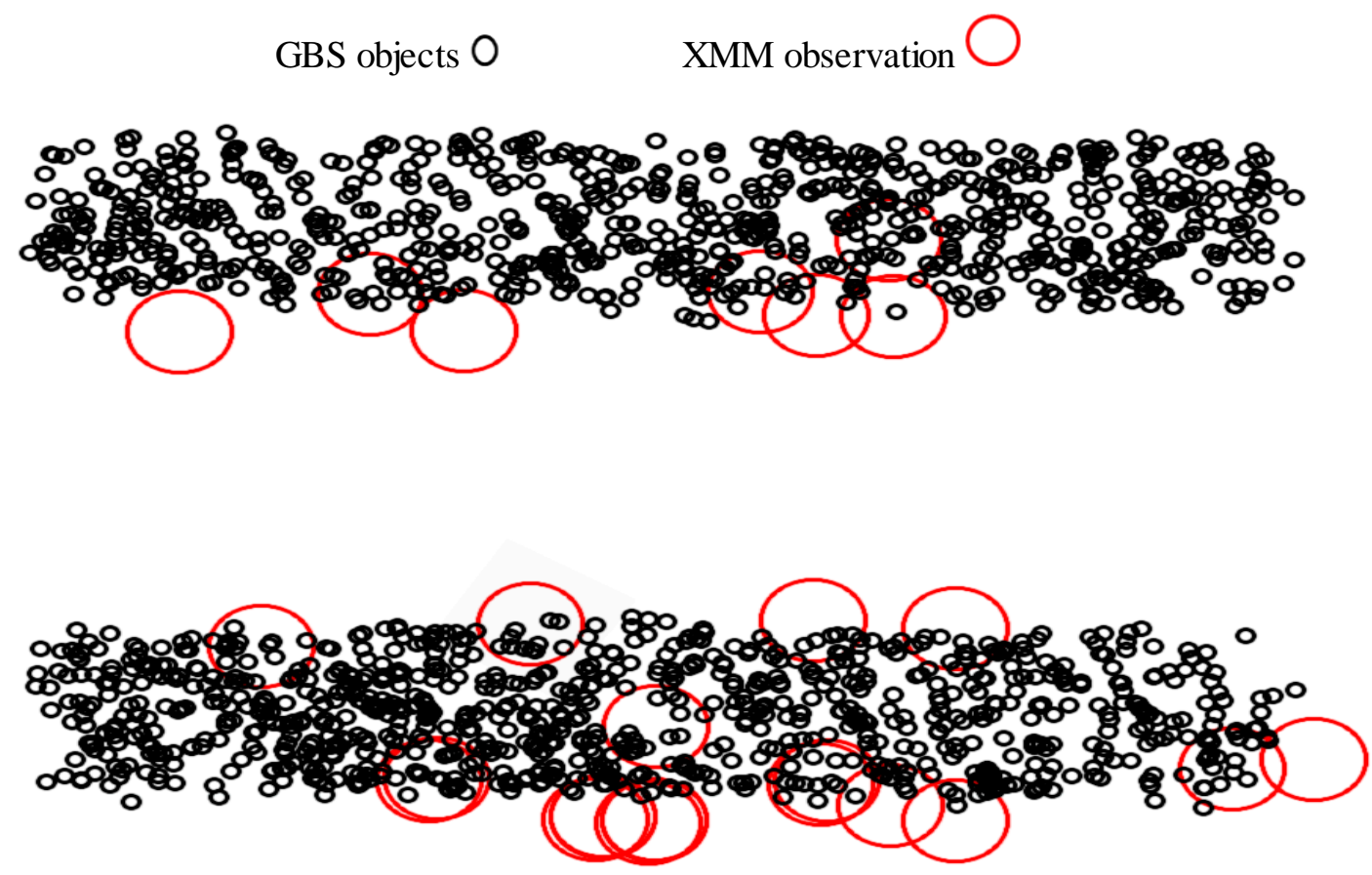

Figure 1: XMM observations overlaid over GBS source detections

\section{Analyzing CX13}

CX13 was the second brightest object in the GBS that was yet to be classified. It has no optical counterpart and is in a region of high extinction, so the XMM data was the only way to classify it. A lower bound on temperature was found using thermal models, which placed it outside the range of an active star. CX5 [1], a confirmed intermediate polar, and CX2 [2], a confirmed background AGN, were also examined to compare with CX13. A power-law model was the best fit to the data and places it between these two options. The power spectrum of each target was constructed to aid in classification. While an AGN may vary in X-rays, the variation is not periodic in the manner seen in the CX2 power spectrum graph. CX5 has a confirmed period, though the XMM data is too noisy to detect it. The CX13 data is also too noisy, though 
there does seem to be a period. Therefore, classification as an absorbed magnetic cataclysmic variable appears to be the most likely interpretation.

CX13
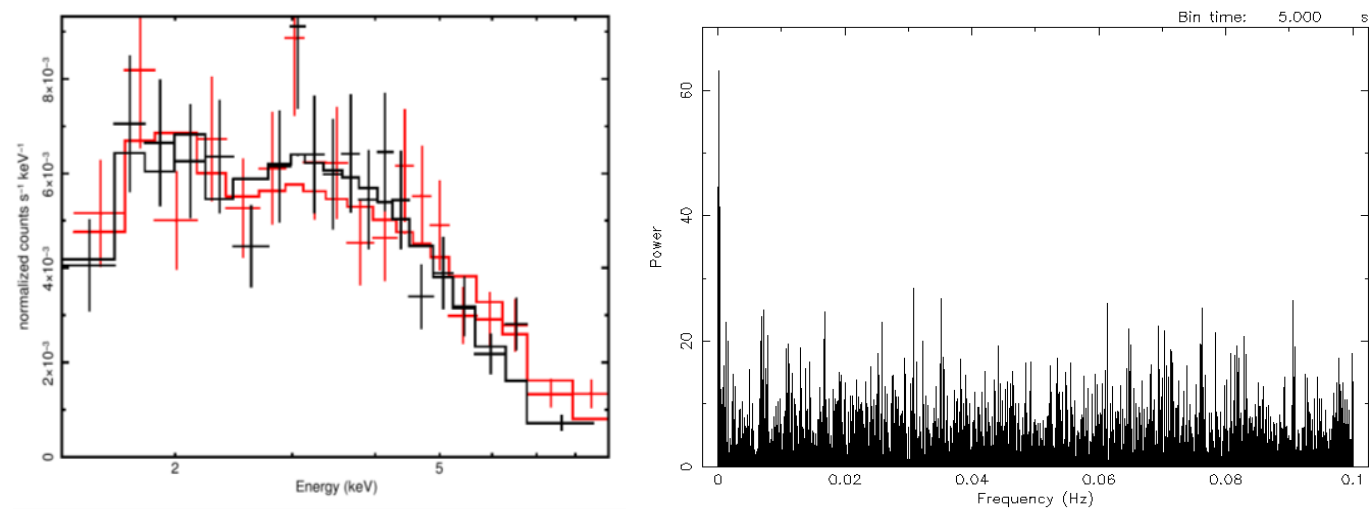

CX5
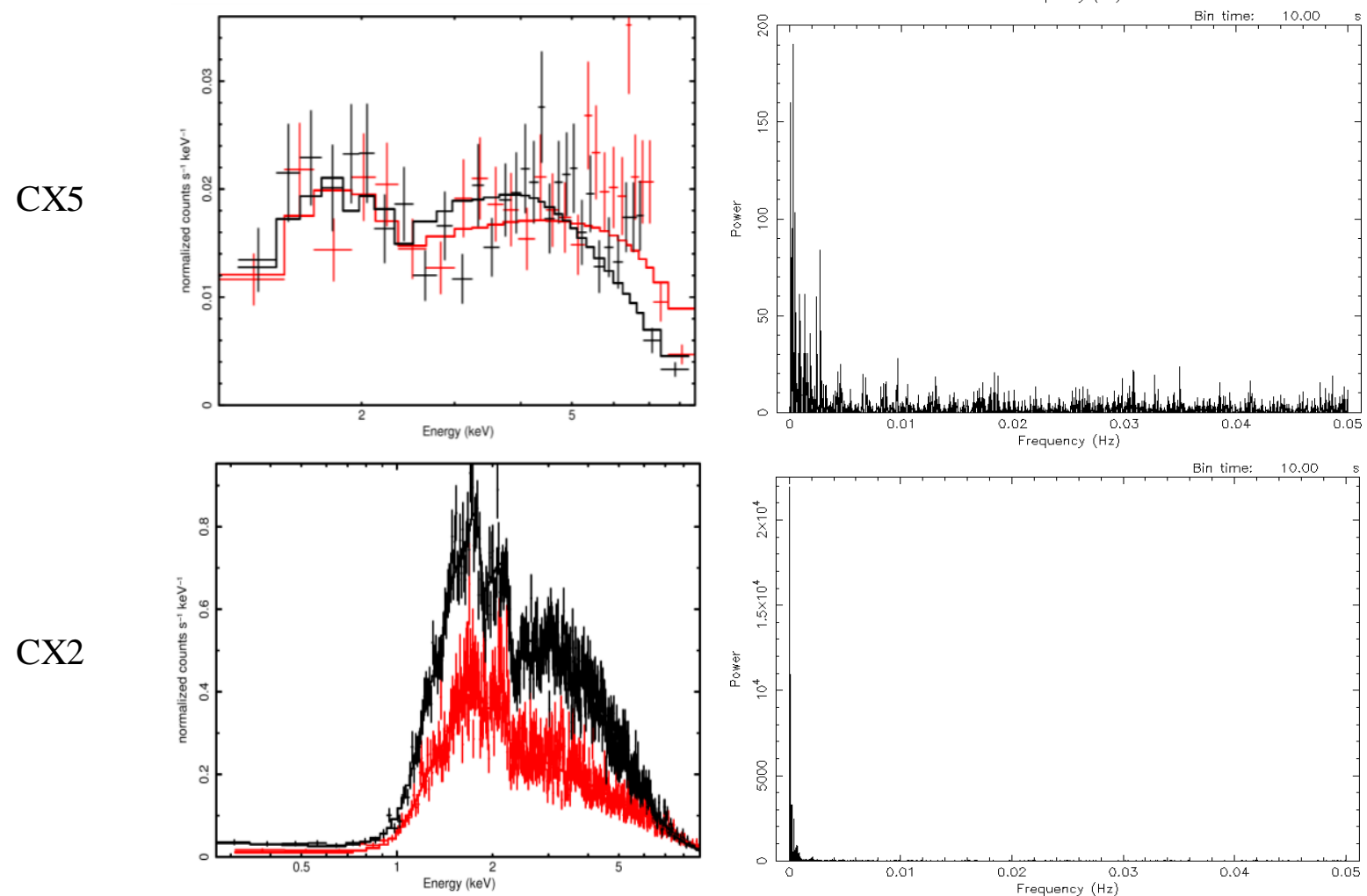

Figure 2: neutral absorber and power-law model fit in left column and power spectrum in right column

\section{References}

[1] C. T. Britt et al., Identification of Five Interacting Binaries in the Galactic Bulge Survey, Astrophysical Journal (2013)

[2] T. J. Maccarone et al., Radio sources in the Chandra Galactic Bulge Survey, Astronomical Society (2012) 